\title{
BMJ Open Evaluation of three-dose fosfomycin tromethamine in the treatment of patients with urinary tract infections: an uncontrolled, open-label, multicentre study
}

\author{
Lu-Dong Qiao, ${ }^{1}$ Bo Zheng, ${ }^{2}$ Shan Chen, ${ }^{1}$ Yong Yang, ${ }^{3}$ Kai Zhang, ${ }^{4}$ \\ Hong-Feng Guo, ${ }^{5}$ Bo Yang, ${ }^{6}$ Yuan-Jie Niu, ${ }^{7}$ Yi Wang, ${ }^{8}$ Ben-Kang Shi, ${ }^{9}$ \\ Wei-Min Yang, ${ }^{10}$ Xiao-Kun Zhao, ${ }^{11}$ Xiao-Feng Gao, ${ }^{12}$ Ming Chen ${ }^{13}$
}

To cite: Qiao L-D, Zheng B, Chen $\mathrm{S}$, et al. Evaluation of three-dose fosfomycin tromethamine in the treatment of patients with urinary tract infections: an uncontrolled, open-label, multicentre study. BMJ Open 2013:3:e004157.

doi:10.1136/bmjopen-2013004157

- Prepublication history for this paper is available online To view these files please visit the journal online (http://dx.doi.org/10.1136/ bmjopen-2013-004157).

LDQ and BZ contributed equally.

Received 1 October 2013 Revised 12 November 2013 Accepted 13 November 2013

CrossMark

For numbered affiliations see end of article.

Correspondence to Professor Shan Chen; shanchentr001@163.com

\section{ABSTRACT}

Objective: To evaluate the clinical and microbiological efficacy and safety of three doses of $3 \mathrm{~g}$ fosfomycin tromethamine administered orally to treat lower urinary tract infections.

Design and participants: This prospective, uncontrolled, open-label study was conducted in 12 medical centres in China, between January and December 2011. According to the diagnosis criteria of Chinese Guidelines on Urological Infections, patients (18-70 years) with acute uncomplicated cystitis, recurrent lower urinary tract infection or complicated lower urinary tract infection received three doses of $3 \mathrm{~g}$ fosfomycin tromethamine orally, at days 1,3 and 5 .

\section{Primary and secondary outcome measures:}

Efficacy endpoints (clinical efficacy, microbiological efficacy and overall efficacy) were evaluated on day 15. Clinical symptoms, physical signs, urinalysis, liver and kidney function, patient records and evaluation of adverse events (AEs) and serious AEs up to day 15 were evaluated for analysis of safety.

Results: 361 patients were included in the full analysis set, 356 in the safety analysis set and 335 in the per-protocol set (PPS). In the PPS, the clinical efficacy rates at day 15 for acute uncomplicated cystitis, recurrent lower urinary tract infection and complicated lower urinary tract infection were $94.71 \%$ (179/189), $77.22 \%$ (61/79) and 62.69\% (42/67), respectively. The microbiological efficacy rates (day 15$)$ were $97.65 \%$ (83/85), $94.44 \%$ (34/36) and $83.87 \%$ (26/31), respectively. The overall efficacy rates (day 15 ) were $95.29 \%$ (81/85), $77.78 \%(28 / 36)$ and $64.52 \%$ (20/31), respectively. 20/356 (5.6\%) patients reported drug-related AEs, the most common being diarrhoea. No serious drug-related AEs were reported.

Conclusions: This fosfomycin tromethamine dosing regimen showed clinical and microbiological efficacy with some AEs and good tolerability in patients with acute uncomplicated cystitis, recurrent lower urinary tract infection and complicated lower urinary tract infection.

\section{Strengths and limitations of this study}

To the best of our knowledge, this is the first multicentre study to evaluate fosfomycin tromethamine in the treatment of lower urinary tract infections (UTIs) in China.

- We used the same dosage regimen for the different types of UTI. The dosage may not be sufficient for the complicated lower UTI, but may exceed the required dose for acute uncomplicated cystitis. Further studies using different dosing regimens for the different types of infection are needed.

- As we aimed to evaluate the clinical, microbiological and overall efficacy of fosfomycin tromethamine, the study design did not include a control group.

\section{INTRODUCTION}

Urinary tract infections (UTIs) are a common clinical infectious disease. Every year, there are more than seven million outpatients and about one million patients are hospitalised due to UTIs. ${ }^{1}$ Death caused by septic shock due to UTIs ranks third among all lethal infections. ${ }^{2}$ In China, UTIs account for $9.39-50 \%$ of all nosocomial infections. ${ }^{34}$

In recent years, many resistant strains have emerged as a consequence of the use of broad-spectrum cephalosporins. In particular, strains producing extended-spectrum $\beta$ lactamase (ESBL) have created a considerable obstacle in the clinical treatment of UTIs. The fosfomycin derivative fosfomycin tromethamine, a broad-spectrum bactericidal antibiotic inhibiting the synthesis of the bacterial cell wall, has been able to maintain resistance rates among Escherichia coli as low as $1 \%$ worldwide. ${ }^{5}$ The available evidence 
suggests that, even for ESBL-producing $E$ coli, the resistance rate to fosfomycin can be maintained at 3.4-16.1\%. ${ }^{6}$ The increasing difficulties associated with the growing drug resistance against other oral antibiotics make fosfomycin even more valuable in the treatment of UTIs. In order to obtain a deeper understanding of its therapeutic effect in domestic patients, we evaluated the clinical and microbiological efficacy and safety in patients with lower UTI from 12 research centres in China.

\section{METHODS}

\section{Study design and participants}

Eligible patients were between 18 and 70 years of age and had sought medical help for acute uncomplicated cystitis (AUC), recurrent lower UTI or complicated lower $\mathrm{UTI}^{8}$ in a urology department of 1 of 12 medical centres in China from January 2011 to December 2011. All study participants provided informed consent.

The main exclusion criteria were: a history of allergy to fosfomycin; susceptibility results showing resistance to the test drug; fever; grade 3 or 4 cardiac insufficiency; pregnant or lactating women, or women of childbearing age with a positive urine pregnancy test; acute pyelonephritis or acute episode of chronic pyelonephritis; previously diagnosed renal failure; liver disease, with alanine transaminase (ALT) or aspartate aminotransferase (AST) greater than two times the upper limit of normal range; white cell count less than $2 \times 10^{9} / \mathrm{L}$; receipt of other anti-infective drugs within $72 \mathrm{~h}$ before enrolment; patients in the late stages of malignant tumours; long-term use of hormones or immunosuppressants; a history of epilepsy or central nervous system disorders; severe diarrhoea; participation in another clinical trial within 3 months prior to enrolment.

\section{Test drug and dosing regimen}

The test drug was fosfomycin tromethamine powder $(3 \mathrm{~g}$ fosfomycin/bottle), produced by Shanxi C\&Y Pharmaceutical Co, Ltd, batch number-20100626.

The treatment duration was 5 days: on the first day (day 1), a dose of $3 \mathrm{~g}$ fosfomycin tromethamine powder was dissolved with an appropriate amount of water and taken on an empty stomach before dinner; two further doses of $3 \mathrm{~g}$ fosfomycin tromethamine powder were taken orally in the evening of days 3 and 5, before bedtime.

\section{Clinical observations}

Infection signs and symptoms, including frequent, urgent, painful and difficult urination, were noted on days 1,8 and 15 .

Blood count, including white blood cells, red blood cells, platelets, neutrophils, lymphocytes, eosinophils and haemoglobin, was performed on days 1 and 8 . Patients with abnormal levels on day 8 were re-examined on day 15.
Urinalysis, including urine proteins and red and white blood cells, was performed on days 1, 8 and 15 .

Blood biochemistry, including liver functions (ALT, AST, $\gamma$-glutamyltransferase, total bilirubin and direct bilirubin) and renal functions (blood urea nitrogen and creatinine), was assessed on days 1 and 8 . Patients with abnormal levels on day 8 were re-examined on day 15 .

Bacterial cultures were obtained for days 1, 8 and 15, using aseptic techniques to collect midstream specimens of urine.

\section{Evaluation of efficacy and safety}

Clinical efficacy, microbiological efficacy, overall efficacy and safety were evaluated on day 15 .

\section{Evaluation of clinical efficacy}

The clinical effect on the lower UTIs of participants was described using the following three grades: cured (symptoms and signs disappeared completely), improved (symptoms and signs improved markedly) and ineffective (symptoms and signs worsened or had no significant improvement). Both 'cured' and 'improved' were considered 'effective' results and were used to calculate the clinical efficacy rate.

\section{Evaluation of microbiological efficacy}

The microbiological efficacy for each patient with a positive urine culture was evaluated from day 1 . We assessed the microbiological effect using the following four grades: eradication (no pathogenic bacteria in the urine were present on days 8 and 15), persistence (pathogenic bacteria were still present on days 8 and 15 or the patient had a negative culture on day 8 , but positive for the same bacteria on day 15), replacement (new pathogenic bacteria were detected on days 8 and 15 , but the patient had no clinical symptoms and no treatment was necessary) and reinfection (new pathogenic bacteria were detected on days 8 and 15; the patient had symptoms and treatment was necessary). Microbiological test results showing 'eradication', 'replacement' or 'reinfection' were considered 'effective', and were used to calculate the microbiological efficacy rate.

\section{Evaluation of overall efficacy}

Overall efficacy was assessed on patients for whom clinical and microbiological efficacy data were available by day 15. Four grades were used: excellent (all assessments (clinical symptoms, physical signs, laboratory tests and bacteriological examinations) had returned to normal by day 15), good (the condition improved markedly, but one of the described assessments remained out of the normal range), fair (some improvement was observed after treatment, but two of the described assessments failed to return to normal) and poor (no significant improvement was observed or the assessments worsened). Cases graded as 'excellent' or 'good' were considered 'effective' results, and were used to calculate the overall efficacy rate. 


\section{Evaluation of safety}

Clinical symptoms, physical signs, urinalysis, liver and kidney function, all patient records and evaluation of adverse events (AEs) and serious AEs were considered for the safety evaluation. According to their severity, AEs were categorised by patients as follows: 'no discomfort', 'mild discomfort', 'moderate discomfort' and 'severe discomfort'. The association between AEs and the study drug was assessed by the physician as 'definitely related', 'probably related', 'possibly related', 'possibly not related' and 'definitely not related'.

\section{Statistical methods}

The data management software EpiData was used for data entry (Lauritsen JM, EpiData Data Entry, Odense Denmark, EpiData Association, 2000-2008. Available from http://www.epidata.dk). Each case report form was completed by two persons and entry results were compared using SAS V.9.2. The verified data were then entered into a database that was considered as final and correct. For efficacy evaluation, the efficacy rates were calculated as a ratio between the 'effective' cases and the total number of patients. For safety analyses, the number of patients with AEs, drug-related AEs and the incidence of AEs were calculated.

The populations included in the analyses were handled as follows: all selected patients who received the study drug were included in the full analysis set (FAS). The per-protocol set (PPS) included the patients from FAS who did not meet the following conditions: (1) inclusion criteria or exclusion criteria violation; (2) use of prohibited concomitant medications; (3) administration of the study drug less than three times; (4) failure to undergo the examinations required to determine the clinical effect; and (5) poor compliance with the study drug. The PPS population was considered as the primary analysis population. All patients who received at least one dose of the study drug and had data available for all safety endpoints were included in the safety analysis set (SS).

\section{RESULTS \\ Baseline demographics}

Three hundred and sixty-one patients enrolled at 12 centres were included in the FAS. Five patients did not take the test drugs and were excluded, resulting in 356 patients being included in the SS. Twenty-one patients were further excluded due to failure to undergo the examinations required to determine the clinical effect, resulting in 335 patients being included in the PPS. Demographic data and infection types for FAS and PPS are shown in table 1 .

\section{Clinical efficacy}

On day 15, the fosfomycin tromethamine treatment showed the clinical efficacy in 282/335 patients. Of these, the symptoms and signs of 228/335 (65.07\%) patients were graded as 'cured'. The clinical efficacy rates for patients of different gender, age or infection types are shown in table 2.

\section{Microbiological efficacy}

One hundred and ninety-eight of 356 patients had positive cultures from urine samples collected on day 1, before receiving the first dose of treatment. The causative pathogens and their resistance profiles will be presented elsewhere.

Microbiological efficacy was determined for 152 patients (46/198 patients did not have urine cultures at follow-up visits). One hundred and twenty-seven of 152 $(83.55 \%)$ patients had a microbiological efficacy grade of 'eradication', 9/152 (5.92\%) patients were graded as 'persistence', 15/152 (9.87\%) patients as 'replacement' and $1 / 152(0.66 \%)$ patients as 'reinfected'. Microbiological outcomes by infection type are shown in table 3. The percentage of patients with an effective

Table 1 Demographic characteristics of patients at enrolment

\begin{tabular}{|c|c|c|c|c|c|}
\hline \multirow[b]{2}{*}{ Variables } & \multirow[b]{2}{*}{ Parameter } & \multicolumn{2}{|l|}{ FAS } & \multicolumn{2}{|l|}{ PPS } \\
\hline & & $\bar{n}$ & Per cent & $\bar{n}$ & Per cent \\
\hline \multirow[t]{2}{*}{ Gender } & Male & 114 & 31.58 & 105 & 29.08 \\
\hline & Female & 247 & 68.42 & 230 & 63.71 \\
\hline Age (years) & & $49.63 \pm 16.64$ & $49.91 \pm 16.61$ & & \\
\hline Height (cm) & & $163.55 \pm 7.12$ & $163.47 \pm 7.05$ & & \\
\hline Weight (kg) & & $61.36 \pm 10.2$ & $61.44 \pm 9.81$ & & \\
\hline Antibiotics* & & 45 & 12.53 & 43 & 12.84 \\
\hline Hypertension & & 65 & 18.01 & 62 & 18.51 \\
\hline Diabetes & & 25 & 6.93 & 24 & 7.16 \\
\hline \multirow[t]{3}{*}{ UTI diagnosis† } & AUC & 204 & 56.51 & 189 & 56.42 \\
\hline & Recurrent lower UTI & 87 & 24.38 & 79 & 23.58 \\
\hline & Complicated lower UTI & 69 & 19.11 & 67 & 20.00 \\
\hline
\end{tabular}




\begin{tabular}{|c|c|c|c|c|}
\hline Variables & $\mathrm{n}$ & Effective, $n$ & Ineffective, n & Efficacy rate (\%) \\
\hline \multicolumn{5}{|l|}{ Gender } \\
\hline Male & 105 & 77 & 28 & 73.33 \\
\hline Female & 230 & 205 & 25 & 89.13 \\
\hline \multicolumn{5}{|l|}{ Age } \\
\hline$<50$ years & 154 & 137 & 17 & 88.96 \\
\hline$\geq 50$ years & 181 & 145 & 36 & 80.11 \\
\hline \multicolumn{5}{|l|}{ Diagnosis } \\
\hline AUC & 189 & 179 & 10 & 94.71 \\
\hline Recurrent lower UTI & 79 & 61 & 18 & 77.22 \\
\hline Complicated lower UTI & 67 & 42 & 25 & 62.69 \\
\hline
\end{tabular}

AUC, acute uncomplicated cystitis; UTI, urinary tract infection.

treatment in terms of microbiological results was $94.08 \%$. For AUC, recurrent lower UTI and complicated lower UTI, the microbiological efficacy by infection type was $97.65 \%$ (83/85), $94.44 \%(34 / 36)$ and $83.87 \%$ (26/31), respectively.

\section{Overall efficacy}

Overall efficacy was assessed for the 152 patients with available microbiological test results. Of these 152 patients, $106(69.74 \%)$ patients were graded as 'excellent', 23/152 (15.13\%) patients as 'good', 14/152 $(9.21 \%)$ patients as 'fair' and 9/152 (5.92\%) patients as 'poor'. The overall efficacy rate by infection type is shown in table 4.

\section{Safety evaluation}

AEs were reported in 20/356 (5.6\%) patients; and the most frequently reported event was diarrhoea (18/356 $(5.06 \%))$. Fourteen patients had mild diarrhoea and 4 had moderate diarrhoea. One patient $(0.28 \%)$ reported mild fatigue and one patient reported mild backache. One patient discontinued the trial due to moderate diarrhoea. All other AEs resolved without further treatment. No patients showed abnormal laboratory test results.

\section{DISCUSSION}

Fosfomycin tromethamine has been used in clinical practice for many years in Europe, but was rarely used in China until recently. To the best of our knowledge, this is the first multicentre study to evaluate fosfomycin tromethamine in the treatment of lower UTIs in this country. It has been previously observed that one dose of $3 \mathrm{~g}$ fosfomycin tromethamine taken orally reaches a peak concentration in the urine with $1053-4415 \mathrm{mg} / \mathrm{L}$ and the fosfomycin concentration in urine is maintained at levels greater than $128 \mathrm{mg} / \mathrm{L}$ for $24-48 \mathrm{~h}$, which is sufficient to suppress a variety of pathogenic bacteria in the urinary tract. ${ }^{9}$ In the current study, patients received three doses of $3 \mathrm{~g}$ fosfomycin tromethamine every other day.

With bacterial resistance rising worldwide, the European Urology Association adjusted its recommendations for the treatment of AUC in the Guidelines on Urological Infections 2010. ${ }^{10}$ The initial recommended first-line drugs, such as sulfamethoxazole trimethoprim (SMZ TMP) and fluoroquinolones, are now recommended as second-choice drugs, while fosfomycin tromethamine is now recommended as the preferred treatment. Stein ${ }^{11}$ made a comprehensive analysis of published randomised controlled clinical studies (MEDLINE 1970-1997) and unpublished studies submitted to the Food and Drug Administration (FDA) on fosfomycin tromethamine treatment of uncomplicated UTI. The results suggested that a single dose of $3 \mathrm{~g}$ fosfomycin tromethamine, taken orally, is superior in terms of compliance and efficacy to conventional therapy (SMZ TMP, nitrofurantoin and fluoroquinolones) administered over 3-7 days to treat uncomplicated UTIs. In line with this, we obtained clinical, microbiological and overall efficacy rates of $94.71 \%, 97.65 \%$ and $95.29 \%$, respectively, for the treatment of AUC using fosfomycin tromethamine; and $77.22 \%, 94.44 \%$ and $77.78 \%$, respectively, for recurrent lower UTI.

Complicated UTIs are associated with conditions such as structural or functional abnormality of the

Table 3 Microbiological outcomes by infection type (152 cases)

\begin{tabular}{lllll}
\hline Diagnosis & $\mathbf{n}$ & Eradication, $\mathbf{n}(\%)$ & Persistence, $\mathbf{n}(\%)$ & Replacement/reinfection, $\mathbf{n}(\%)$ \\
\hline AUC & 85 & $77(90.59)$ & $2(2.35)$ & $6(7.06)$ \\
Recurrent lower UTI & 36 & $27(75.00)$ & $2(5.56)$ & $7(19.44)$ \\
Complicated lower UTI & 31 & $23(74.19)$ & $5(16.13)$ & $3(9.68)$ \\
\hline
\end{tabular}

AUC, acute uncomplicated cystitis; UTI, urinary tract infection. 
Table 4 Overall efficacy by infection type (152 cases)

\begin{tabular}{lllll}
\hline Diagnosis & $\mathbf{n}$ & Effective, $\mathbf{n}$ & Ineffective, $\mathbf{n}$ & Efficacy rate (\%) \\
\hline AUC & 85 & 81 & 4 & 95.29 \\
Recurrent lower UTI & 36 & 28 & 8 & 77.78 \\
Complicated lower UTI & 31 & 20 & 11 & 64.52 \\
\hline AUC, acute uncomplicated cystitis; UTI, urinary tract infection. & &
\end{tabular}

genitourinary tract, or the presence of an underlying disease that interferes with host defence mechanisms, increasing the risks of acquiring infection or of failing therapy. Complicated UTI is caused by a broader spectrum of pathogenic bacteria, and is associated with a higher level of drug resistance, than uncomplicated UTI. Domestic research has shown that the isolation rate for ESBL-producing $E$ coli is $44 \%$ in complicated UTI, with susceptibility data showing that ESBL-producing strains have significantly higher resistance against commonly used antibiotics than the non-ESBL-producing strains. $^{12} 13$ With a growing proportion of ESBL-producing strains identified in UTIs, the resistance rate of $E$ coli to commonly used second-generation and third-generation cephalosporins has increased to more than $45 \% .^{14}$ This creates considerable obstacles for effective antimicrobial use in clinical practice. As a direct result, carbapenem has been increasingly used in the empirical treatment of complicated UTI, which has led to an increase in bacterial resistance, an imbalance of commensal flora and increased risk of fungal infection. ${ }^{15}$ Fortunately, unlike resistance against cephalosporins and fluoroquinolones of common UTI pathogens, the resistance rate to fosfomycin has not increased in the recent years. ${ }^{16}$ Previous single-centre studies have achieved good results with fosfomycin in the treatment of UTIs caused by ESBL-producing bacteria. ${ }^{17}$ In line with this, the current study showed clinical, microbiological and overall efficacy rates of $62.69 \%, 83.87 \%$ and $64.52 \%$, respectively, in the treatment of complicated lower UTI.

We observed a low frequency of AEs in the current study. Our findings are in line with an overview of safety and tolerability results from 12 open and double-blind comparative studies, ${ }^{18}$ in which 891 female patients received a single $3 \mathrm{~g}$ dose of fosfomycin tromethamine. In that study, AEs were reported for $6.1 \%$ of the patients, with gastrointestinal disorders being the most frequent.

One limitation of this study results from the same dosage regimen (3 doses, of $3 \mathrm{~g}$ each, of fosfomycin tromethamine taken orally) used for the different types of UTI. The dosage may not be sufficient for the complicated lower UTI, but may exceed the required dosage for simple AUC. Therefore, further in-depth studies will be needed, with different treatment regimens for the different types of infection. Another shortcoming of the study is the lack of a control group. This is due to the fact that our primary objective was to evaluate the clinical, microbiological and overall efficacy of fosfomycin tromethamine, an antibiotic which is widely used abroad, but less in China, for the treatment of UTI.

\section{CONCLUSIONS}

The current study suggests that three single $3 \mathrm{~g}$ doses of fosfomycin tromethamine taken orally, every other day, are effective and well tolerated, with a clinically acceptable safety profile in the treatment of AUC, recurrent lower UTI or complicated lower UTI. We believe that this dosage regimen can be adopted to treat lower UTIs in clinical practice.

\section{Author affiliations}

${ }^{1}$ Department of Urology, Beijing Tongren Hospital Capital Medical University, Beijing, People's Republic of China

${ }^{2}$ Institute of Clinical Pharmacology, Peking University First Hospital, Beijing, People's Republic of China

${ }^{3}$ Department of Urology, Beijing Cancer Hospital, Beijing, People's Republic of China

${ }^{4}$ Department of Urology, Peking University First Hospital, Beijing, People's Republic of China

${ }^{5}$ Department of Urology, Peking University Shougang Hospital (Wu Jieping Urology Center), Beijing, People's Republic of China

${ }^{6}$ Department of Urology, Peking University People's Hospital, Beijing, People's Republic of China

${ }^{7}$ Department of Urology, Second Affiliated Hospital of Tianjin Medical University, Tianjin, People's Republic of China

${ }^{8}$ Department of Urology, First Affiliated Hospital of China Medical University, Shenyang, People's Republic of China

${ }^{9}$ Department of Urology, Qilu Hospital of Shandong University, Jinan, People's Republic of China

${ }^{10}$ Department of Urology, Wuhan Hubei Tongji Hospital, Wuhan, People's Republic of China

${ }^{11}$ Department of Urology, Hunan Province Xiangya Second Hospital, Changsha, People's Republic of China

${ }^{12}$ Department of Urology, Shanghai Changhai Hospital, Shanghai, People's Republic of China

${ }^{13}$ Department of Urology, Zhongda Hospital Southeast University, Nanjing, People's Republic of China

Acknowledgements The authors would like to thank all study participants and the general practitioners, study nurses and personnel who contributed to this study. The authors wish to acknowledge the support provided by Linyu $\mathrm{Li}$ and Jinghan Zhang. They also thank Juliette Gray and Adriana Rusu (XPE Pharma \& Science) for editorial support.

Contributors SC, L-DQ and BZ designed the research and defined the research theme; L-DQ, YY, KZ, H-FG, BY, Y-JN, YW, B-KS, W-MY, X-KZ, X-FG and $M C$ performed the research. SC and L-DQ analysed the data and interpreted the results. All authors contributed to the writing of the manuscript and have read and approved the final version of the manuscript.

Funding This research received no specific grant from any funding agency in the public, commercial or not-for-profit sectors.

Competing interests None. 
Patient consent Obtained.

Ethics approval This was a prospective, uncontrolled, open-label, multicentre study approved by the ethics committee of the Beijing Tongren Hospital.

Provenance and peer review Not commissioned; externally peer reviewed.

Data sharing statement No additional data are available.

Open Access This is an Open Access article distributed in accordance with the Creative Commons Attribution Non Commercial (CC BY-NC 3.0) license, which permits others to distribute, remix, adapt, build upon this work noncommercially, and license their derivative works on different terms, provided the original work is properly cited and the use is non-commercial. See: http:// creativecommons.org/licenses/by-nc/3.0/

\section{REFERENCES}

1. Foxman B. Epidemiology of urinary tract infections: incidence, morbidity, and economic costs. Am J Med 2002;113:5S-13S.

2. Pinner RW, Teutsch SM, Simonsen $L$, et al. Trends in infectious disease mortality in the United States. JAMA 1996;275:189-93.

3. Wang JQ, Wu XP, Li YJ, et al. Nosocomial infection point-prevalence survey in three consecutive years. Chin $\mathrm{J}$ Infect Control 2006;01:19-34.

4. Wang JX, Zhu XL, Yang LY. A nosocomial infection point-prevalence survey: results and analysis. Chin J Nosocomiol 2005;10:1119-20.

5. Schito GC. Why fosfomycin trometamol as first line therapy for uncomplicated UTI? Int J Antimicrob Agents 2003;22:79-83.

6. Qi J, Cheng X. Results analysis of ESBLs-producing E. coli in vitro susceptibility to fosfomycin. China Trop Med 2010;10:734-5.

7. Da PN, Rong M, Yong EL. In vitro antimicrobial activity of fosfomycin against Gram-negative bacilli. Dalian Med Univ 2010;32:577-9.
8. $\mathrm{Na} Y \mathrm{Y}$, Sun G. Guidelines for diagnosis and treatment of urological diseases in China, 2009. Beijing: People's Health Publishing House, 2009:643-4.

9. Patel SS, Balfour JA, Bryson HM. Fosfomycin tromethamine. A review of its antibacterial activity, pharmacokinetic properties and therapeutic efficacy as a single-dose oral treatment for acute uncomplicated lower urinary tract infections. Drugs 1997;53:637-56.

10. Grabe M, Bjerklund-Johansen TE, Botto $\mathrm{H}$, et al. European Association of Urology, Guidelines on Urological Infections. 2010. http://www.uroweb.org/

11. Stein GE. Single-dose treatment of acute cystitis with fosfomycin tromethamine. Ann Pharmacother 1998;32:215-19.

12. Qiao LD, Chen S. Analysis of risk factors about ESBLs-producing E. coli urinary tract infection. J Hosp Infect 2011;21:247-9.

13. Kong HS, Wang BG. Comparative study of antibiotic resistance in clinical isolates of Klebsiella pneumoniae and Escherichia coli producing extended-spectrum beta lactamases. J Lab Med 2000;23:23-5.

14. Gao L, Xiao YH. 2006-2007 Mohnarin report: bacterial distribution and resistance in urinary tract infections. Chin $J$ Antibiot 2008;33:586-91.

15. Chen H, Liu Y. Relationship between antimicrobial treatment and imbalance of intestinal flora in the elders. Pract Clin Med 2007;9:34-6.

16. Pullukcu $\mathrm{H}$, Aydemir S, Tasbakan $\mathrm{M}$, et al. Is there a rise in resistance rates to fosfomycin and other commonly used antibiotics in Escherichia coli-mediated urinary tract infections? A perspective for 2004-2011. Turk J Med Sci 2013;43:537-41.

17. Pullukcu H, Tasbakan M, Sipahi OR, et al. Fosfomycin in the treatment of extended spectrum beta-lactamase-producing Escherichia coli-related lower urinary tract infections. Int J Antimicrob Agents 2007;29:62-5.

18. Naber KG. Fosfomycin trometamol in treatment of uncomplicated lower urinary tract infections in adult women-an overview. Infection 1992;20:S310-12 